\title{
Modelling of Flow Stress and Prediction of Workability by Processing Map for Hot Compression of 43CrNi Steel
}

\author{
Niranjan KUMAR, ${ }^{1)}$ Sanjeev KUMAR, ${ }^{1)}$ Sunil Kumar RAJPUT ${ }^{21}$ and Sumeer Kumar NATH ${ }^{1 / *}$ \\ 1) Department of Metallurgical and Materials Engineering, Indian Institute of Technology, Roorkee, 247667 India. \\ 2) Department of Mechanical Engineering, Bundelkhand Institute of Engineering and Technology, Jhansi, 284128 India.
}

(Received on May 21, 2016; accepted on November 16, 2016)

\begin{abstract}
The isothermal hot compression tests of $43 \mathrm{CrNi}$ steel was carried out at temperatures $800^{\circ} \mathrm{C}$ to $1050^{\circ} \mathrm{C}$ at an interval of $50^{\circ} \mathrm{C}$ and at constant strain rates of $0.01,0.1,1.0$ and $10 \mathrm{~s}^{-1}$ for total true strain of 0.7 . The values of experimental stress were corrected for adiabatic heating which was used for flow stress modeling, and further its predictability was verified with experimental results. The true stress-true strain curves exhibit peak stresses followed by softening due to occurrence of DRX. The activation energy was calculated based on sinh type equation and found to be increasing with increase in strain. The obtained stress exponent 5.4, suggests that the mechanism of hot deformation is dislocation glide controlled by dislocation climb. Strain rate sensitivity maps and processing maps were developed using dynamic materials model (DMM), modified DMM with different instability criteria. The instability parameter, $\kappa_{j}$, is directly related to the dissipative function which is associated to microstructural changes, $J$, whereas the instability parameters, $\xi$ and $\kappa$, are associated with the strain rate sensitivity parameter, $m$. Therefore, the instability parameter, $\kappa_{j}$, is more mathematically precise as it eliminates $m$ from its calculations. The microstructures of deformed specimens were studied and correlated with the domains of processing maps to validate the workability region.
\end{abstract}

KEY WORDS: medium carbon low alloy steel; processing maps; hot deformation; constitutive analysis; thermo mechanical simulator.

\section{Introduction}

Application of low alloy medium carbon steel is continuously increasing due to its very good strength, toughness, wear resistance, and excellent property of forged products. These steels are widely used for making shafts, shaft gears, spindles, rams, crow bars, ring gears, connecting rods, crankshafts, wheel hubs, railway wheels, high tensile tubing and high tensile fasteners. ${ }^{1,2}$ To achieve the high strength and toughness, grain refinement and microstructural control of hot rolled, forged and extruded product are necessary. In order to improve the final properties of the hot worked product, the process parameters must be optimized. Various metallurgical phenomena occur in metals during hot working, which leads the complex microstructural evolutions. Therefore, the knowledge of rate controlling mechanism and the effect of strain rate on hot deformation behavior are required.

A number of studies on medium carbon steel have been carried out on thermo-mechanical simulator to develop the processing maps and correlate its domain with evolved microstructures. This helps to optimize hot deformation parameters i.e. strain, strain rate and temperature. Physical simulation filled the gap between the mathematical model-

* Corresponding author: E-mail: indiafmt@iitr.ac.in DOI: http://dx.doi.org/10.2355/isijinternational.ISIJINT-2016-306 ing and real world application of metals. Lin et al. established a constitutive relation between processing parameters to predict the flow stress behavior of $42 \mathrm{CrMo}$ steel. ${ }^{3)}$ Hase et al. investigated the effect of initial grain size during warm deformation of medium carbon steels and finally observed the fine ferritic grain size of $0.5 \mu \mathrm{m}$. $\left.{ }^{4}\right)$ Wei et al. observed that carbon addition can decrease the work hardening rate at low strain rate and accelerate the dynamic recrystallization of vanadium micro-alloyed medium carbon steel. ${ }^{5)}$ Zhao et al. performed isothermal hot compression tests on vanadium micro-alloyed medium carbon steel and observed that the activation energy of hot working at low strain rates is very close to the activation energy of austenite lattice selfdiffusion. ${ }^{2)}$ Rajput et al. performed hot compression tests on AISI 1016 steel using thermo-mechanical simulator and developed the processing maps based on DMM and modified DMM with different instability criteria. ${ }^{6}$

From the above studies, it is observed that flow stress prediction has been done only for narrow temperature domains. Processing maps were developed based only on dynamic materials model (DMM). No attempt has been made to study modified DMM model and other instability parameters. Very few studies have been done to verify the theoretical model with the experimental results. There has been lack of validation of various instability regimes with evolved microstructures. In view of this, the present study is focused to model the processing parameters to predict the 
flow stress of $43 \mathrm{CrNi}$ steel and to delineate safe and unsafe zone in processing maps based on different models and to obtain an optimum combination of temperature and strain rate at a fixed given strain.

\section{Materials and Experimental Method}

$43 \mathrm{CrNi}$ steel is procured in the form of cylindrical rod of diameter $10 \mathrm{~mm}$. The chemical composition of this steel is determined with the help of Thermo Jarrell Ash spark emission spectroscope and is given in Table $\mathbf{1}$.

The cylindrical hot compression and dilatometry tests specimens were machined from steel rod of dimensions $\phi 10 \mathrm{~mm} \times 15 \mathrm{~mm}$ length and $\phi 10 \mathrm{~mm} \times 70 \mathrm{~mm}$ length, respectively. The compression tests were performed on Gleeble $^{\circledR} 3800$ thermo-mechanical simulator under vacuum (pressure of $1 \mathrm{~Pa}$ ). AK-type thermocouple was spot welded with the help of spot welding machine at the longitudinal center of the specimen to monitor the temperature during the compression test. To reduce friction and temperature gradient, a 10 mil graphite foil and a nickel-based lubricant were used between the specimen and ISO-T anvil. Each specimen was heated with heating rate of $5^{\circ} \mathrm{Cs}^{-1}$ up to austenitization temperature of $1100^{\circ} \mathrm{C}$ and soaked for $2 \mathrm{~min}$ and followed by cooling with $1^{\circ} \mathrm{Cs}^{-1}$ to deformation temperatures ranging from $800-1050^{\circ} \mathrm{C}$ with an interval of $50^{\circ} \mathrm{C}$ as shown in Fig. 1(a). The photograph of specimen before and after deformation is shown in Fig. 1(b). The justification of selecting austenitization temperature $1100^{\circ} \mathrm{C}$ is because chromium carbide $\left(\mathrm{Cr}_{23} \mathrm{C}_{6}\right.$ and $\left.\mathrm{Cr}_{7} \mathrm{C}_{3}\right)$ begins to dissolve at $900^{\circ} \mathrm{C}$ and is completely in solution at $1100^{\circ} \mathrm{C}^{7)}$ The compression tests were performed at four constant strain rates i.e. $0.01,0.1,1$ and $10 \mathrm{~s}^{-1}$ upto total true strain of 0.7 . All specimens were in-situ water quenched after the deformation to freeze the microstructure. Finally, simulated specimens are sectioned centrally parallel to the compression axis, and prepared for metallography using ASTM E3-11 standard procedure. All the specimens were etched by $2 \%$ nital for optical microscopy and scanning electron microscopy. The abluent solution of saturated picric acid was used to reveal the prior austenite grain boundary.

Table 1. Chemical composition of present steel used (wt.\%).

\begin{tabular}{cccccccccc}
\hline $\mathrm{C}$ & $\mathrm{Si}$ & $\mathrm{Mn}$ & $\mathrm{P}$ & $\mathrm{S}$ & $\mathrm{Cr}$ & $\mathrm{Ni}$ & $\mathrm{Al}$ & $\mathrm{Cu}$ & $\mathrm{Fe}$ \\
\hline 0.43 & 0.27 & 0.21 & 0.01 & 0.002 & 0.89 & 0.80 & 0.01 & 0.16 & Bal. \\
\hline
\end{tabular}

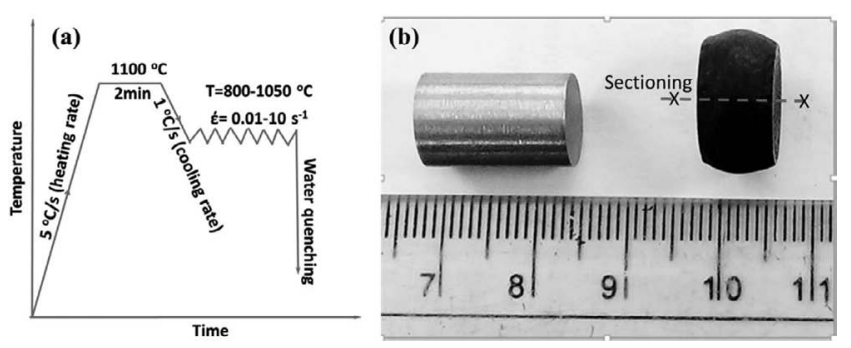

Fig. 1. (a) A schematic diagram showing thermo-mechanical processing cycle and (b) photograph of specimen before and after deformation.

\section{Basic Theory of Processing Maps}

\subsection{Dynamic Materials Model}

Dynamic materials model (DMM) was proposed by Prasad et al. to analyze the hot deformation with following objectives: ${ }^{8)}$

(a) To determine the microstructural response of the specimen in the axes of processing parameters.

(b) To incorporate the behavior of materials with the continuum approach that simulates metal-forming processes using mechanics of large plastic flow.

This model considers the mechanical processing as a system, and the hot deformed specimen as fully dissipater of the total input power $(P)$. The total input power is divided into two parts i.e. the sum of two integrals as given by Eq. (1), whereas the strain rate sensitivity $(m)$, is given by Eq. (2). Most of the energy dissipated as temperature rise given by the first integral ( $G$ content) and rest is dissipated due to microstructural changes, as given by second integral $(J$ co-content). The total power dissipation leads to the entropy generation as given by Eq. (4).

$$
\begin{aligned}
& P=G+J=\int_{0}^{\dot{\varepsilon}} \sigma d \dot{\varepsilon}+\int_{0}^{\sigma} \dot{\varepsilon} d \sigma=\sigma \dot{\varepsilon} \\
& \left(\frac{\partial J}{\partial G}\right)_{T, \varepsilon}=\frac{\sigma d \dot{\varepsilon}}{\dot{\varepsilon} d \sigma}=\left[\frac{\partial \ln \sigma}{\partial \ln \dot{\varepsilon}}\right]_{T, \varepsilon}=m \\
& G=\int_{0}^{\dot{\varepsilon}} \sigma d \dot{\varepsilon}=\int_{0}^{\dot{\varepsilon}_{\min }} \sigma d \dot{\varepsilon}+\int_{\dot{\varepsilon}_{\min }}^{\dot{\varepsilon}} \sigma d \dot{\varepsilon}=\left[\frac{\sigma \dot{\varepsilon}}{m+1}\right]_{\dot{\varepsilon}=\dot{\varepsilon}_{\min }}+\int_{\dot{\varepsilon}_{\min }}^{\dot{\varepsilon}} \sigma d \dot{\varepsilon} \ldots \\
& P=\bar{\sigma} \overline{\dot{\varepsilon}}=T \frac{d S}{d t} \geq 0
\end{aligned}
$$

The overprinting of two maps, the power dissipation efficiency map $(\eta)$ and the instability map $(\xi(\dot{\varepsilon}))$, at constant strain produces processing map. The power dissipation efficiency $(\eta)$ and instability parameter $(\xi(\dot{\varepsilon}))$ of Prasad et al. are given by Eqs. (5) and (6) respectively. ${ }^{9)}$

$$
\begin{gathered}
\eta_{\text {Prasad }}=\frac{J}{J_{\max }}=\frac{2 m}{(m+1)} \cdots \cdots \\
\xi_{\text {Pr asad }}=\left(\frac{\partial \ln \left(\frac{m}{m+1}\right)}{\partial \ln (\dot{\varepsilon})}\right)+m<0
\end{gathered}
$$

The flow instabilities are related to flow localization, which was suggested by Ziegler, that flow instability occurs when the differential quotient satisfies the inequality as given by Eq. (7). ${ }^{10)}$

$$
\frac{\partial D}{\partial \dot{\varepsilon}}<\frac{D}{\dot{\varepsilon}}
$$

Where, $D$ is the power dissipation function.

\subsection{Modified Dynamic Materials Model}

The efficiency and instability parameter $(\kappa)$ of modified DMM is given by Murty et al. without considering the power law, and are given as: ${ }^{11)}$ 


$$
\begin{aligned}
\eta_{\text {Murty\& Rao }}=\frac{2 J}{P} & =\frac{2(P-G)}{P}=\frac{2\left(1-\int_{0}^{\dot{\varepsilon}} \sigma d \dot{\varepsilon}\right)}{\sigma \dot{\varepsilon}} \ldots \ldots . . . \\
\kappa & =\frac{2 m}{\eta}-1<0
\end{aligned}
$$

\subsection{Poletti Instability Criteria}

Poletti et al. derived a new method of finding instability domain to avoid the necessity of strain rate sensitivity, m, in the calculation of instability parameter, $\kappa_{j}$, which is used by Rajput et al. and is given by Eq. (10). ${ }^{12,13)}$

$$
\kappa_{j}=\left(\frac{\partial \ln J}{\partial \ln \dot{\varepsilon}}-1\right)<0
$$

Where, $J$ represents the power dissipated through microstructural changes in the specimen.

In the present work, the strain rate sensitivity $(m)$ was calculated at fixed temperature and strain (Eq. (2)). The power dissipation efficiency maps are plotted using $\eta_{\text {Prasad }}$ and $\eta_{\text {Murty and Rao }}$ (Eqs. (5) and (8) respectively) in the axes of strain rate and temperature. The various instability parameter viz. $\xi$, $\kappa$, and $\kappa_{j}$ are superimposed on the efficiency map in order to determine the instability domains.

\section{Results and Discussion}

\subsection{Phase Transformation Characterization}

The critical temperatures were determined by dilatometry test using thermo-mechanical simulator, and the temperature is monitored by using $\mathrm{K}$-type thermocouple. The specimen is heated to $1100^{\circ} \mathrm{C}$ with the heating rate of $5^{\circ} \mathrm{Cs}^{-1}$ followed by cooling at the same rate. The dilation in the specimen is recorded with the help of Linear Variable Displacement Transducer (LVDT) quartz dilatometer. As temperature increases, the dilation in the specimen increased up to $752^{\circ} \mathrm{C}$ and then decreased up to $823^{\circ} \mathrm{C}$ due to formation of austenite (contraction occurs as austenite is a close packed structure), further it increased after complete formation of austenite as shown in Fig. 2. When cooling is done from $1100^{\circ} \mathrm{C}$, contraction occurs due to thermal contraction in the specimen until proeutectoid ferrite starts precipitating at temperature just below $\mathrm{A}_{\mathrm{r} 3}$ temperature. Due to the precipitation of pro-eutectoid ferrite which has a open structure (bcc), causes increase in dilation which is depicted by a hump at a temperature $673^{\circ} \mathrm{C}\left(\mathrm{A}_{\mathrm{r} 3}\right)$. As temperature keeps on decreasing more and more pro-eutectoid ferrite precipitates in the austenite matrix till the lower critical temperature $512^{\circ} \mathrm{C}\left(\mathrm{A}_{\mathrm{r} 1}\right)$ (change in slope is observed) after this thermal contraction occurs in the specimen as temperature decreases.

\subsection{Flow Stress Behavior}

The flow curves of specimens obtained by isothermal hot compression test at $0.01 \mathrm{~s}^{-1}, 0.1 \mathrm{~s}^{-1}, 1 \mathrm{~s}^{-1}$, and $10 \mathrm{~s}^{-1}$ strain rate and $800^{\circ} \mathrm{C}, 850^{\circ} \mathrm{C}, 900^{\circ} \mathrm{C}, 950^{\circ} \mathrm{C}, 1000^{\circ} \mathrm{C}$, and $1050^{\circ} \mathrm{C}$ are shown in Fig. 3. For a fixed strain and strain rate, these stresses are corrected for adiabatic temperature rise (dT) by using linear interpolation method between $\ln (\sigma)$

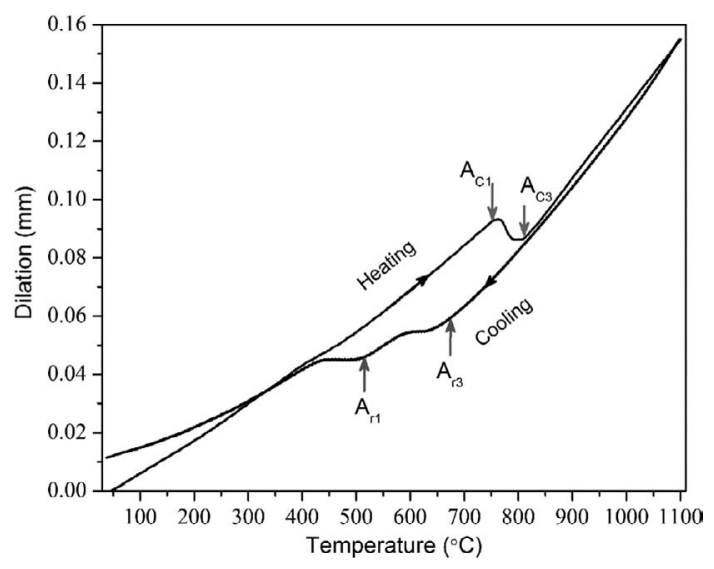

Fig. 2. Dilation curve for the steel showing critical temperatures.

and $1 / T$ and plotted with experimental curves as shown in Fig. 3. ${ }^{14,15)}$ The effect of dT are mostly observed at high strain rates and low temperature. Further, for the analysis of constitutive equations, prediction of flow stress and development of processing maps, the corrected stress values are used.

It can be seen from Fig. 3 that the flow behavior remarkably affected by deformation temperature and strain rates. At strain rate $10 \mathrm{~s}^{-1}$, broad stress peak values are obtained at all respective temperature except $1050^{\circ} \mathrm{C}$ due to dominance of strain hardening, whereas softening is only seen at deformation temperature $1050^{\circ} \mathrm{C}$ followed by steady state. Flow curve which exhibits a steady state is generally associated with dynamic recovery as the dominant restoration mechanism. ${ }^{16)}$ All the tests performed at lower strain rate $\left(0.01 \mathrm{~s}^{-1}\right.$ and $0.1 \mathrm{~s}^{-1}$ at $\left.850-1050^{\circ} \mathrm{C}\right)$ show a peak, followed by softening. The stress peaks shift to higher strain with increasing strain rate at a fixed temperature. Flow curve shows two stress peaks at deformation temperature of $1050^{\circ} \mathrm{C}$ with strain rate of $0.01 \mathrm{~s}^{-1}$, which corresponds to multiple peaks DRX that results in grain growth. ${ }^{13)}$ For a fixed strain rate, as the temperature increases, flow stress decreases gradually, and for a fixed temperature flow stress increases with increasing strain rate as shown in Fig. 4.

\subsection{Constitutive Equations Analysis for Flow Behavior}

To investigate the plastic hot deformation flow behavior, it is essential to study constitutive characteristics. For plastically deforming materials, the steady state flow stress, temperature and strain rate are inter-dependent and the relation is given by power law ${ }^{17)}$ (Eq. (11)).

$$
\bar{\sigma}=k(T, \bar{\varepsilon}, \overline{\dot{\varepsilon}}) \overline{\dot{\varepsilon}}^{m(T, \varepsilon, \dot{\varepsilon})}
$$

The effect of temperature and strain rates on the flow behavior can be given by temperature compensated strain rate called Zener-Hollomon parameter $(Z)$ in Arrhenius type equations (Eqs. (12), (13) and (14)): $:^{3,18)}$

$$
\begin{aligned}
Z= & \dot{\varepsilon} \exp \left(\frac{Q}{R T}\right)=C \sigma^{n^{\prime}} \alpha \sigma<0.8 \ldots \ldots \ldots \ldots . . . \\
& =B \exp (\beta \sigma) \alpha \sigma>1.2 \ldots \ldots \ldots \ldots \ldots \ldots \ldots \ldots \ldots \ldots \ldots
\end{aligned}
$$

where $R$ is the universal gas constant $\left(8.314 \mathrm{~J}\right.$ mole $\left.{ }^{-1} \mathrm{~K}^{-1}\right)$; 
$T$, absolute temperature $(\mathrm{K}) ; Q$, apparent activation energy $\left(\mathrm{KJ} \mathrm{mole}^{-1}\right) ; \dot{\varepsilon}$, the strain rate and $A, B, C, n, n$ ', $\beta$, and $\alpha$ $\left(\alpha=\beta / n^{\prime}\right)$ are the material constants.

\subsubsection{Determination of Material Constants}

The corrected flow stress data at true strain 0.6 are used to analyze Eqs. (12), (13) and (14) to determine apparent activation energy. The value of n', $\beta$ and $\mathrm{n}$ can be obtained by the average slopes of linear fitting of $\ln (\sigma)$, stress and $\ln (\sinh (\alpha \sigma))$ values with respect to $\ln (\dot{\varepsilon})$ at constant temperatures respectively (Figs. 5(a)-5(c)). The analysis yields the value of $n$ ', $\beta$ and $n$ as 7.5774, 0.04999 and 5.4, respectively. From these $\alpha$ is calculated as 0.006598 $\mathrm{MPa}^{-1}$. The average value of slopes of $\ln (\sinh (\alpha \sigma))$ versus $1 / T$ (Fig. 6(a)) at different fixed strain rates is 8235.265 , and the apparent activation energy is calculated as 368.1
$\mathrm{KJmol}^{-1}$ using sinh type law. Similar value of apparent activation energy was reported for medium carbon microalloyed steel. ${ }^{19,20)}$ This activation energy value is much larger than the self -diffusion activation energy of austenitic steel $(311.1 \mathrm{KJ} / \mathrm{mol})$ due to influence of alloying elements having lower stacking fault energy. ${ }^{21)}$ By putting the values of the strain rate, temperatures and flow stress, the relationship between $\ln [\sinh (\alpha \sigma)]$ and $\ln (\mathrm{Z})\left(\mathrm{R}^{2}=0.987\right)$ can be obtained (Fig. 6(b)), whose intercept yields the value of material constant $A$ as $2.619 \times 10^{15} \mathrm{~s}^{-1}$. Among Arrhenius type equations, the sinh type law gives superior estimation between Zener-Hollomon parameter and stress for all range of stresses. ${ }^{1,20)}$ The value of stress exponent $\mathrm{n}$ is 5.4, which suggests that the mechanism of hot deformation is dislocation glide controlled by dislocation climb. ${ }^{22)}$ Finally, substituting all the values of material constants in Eq. (14),
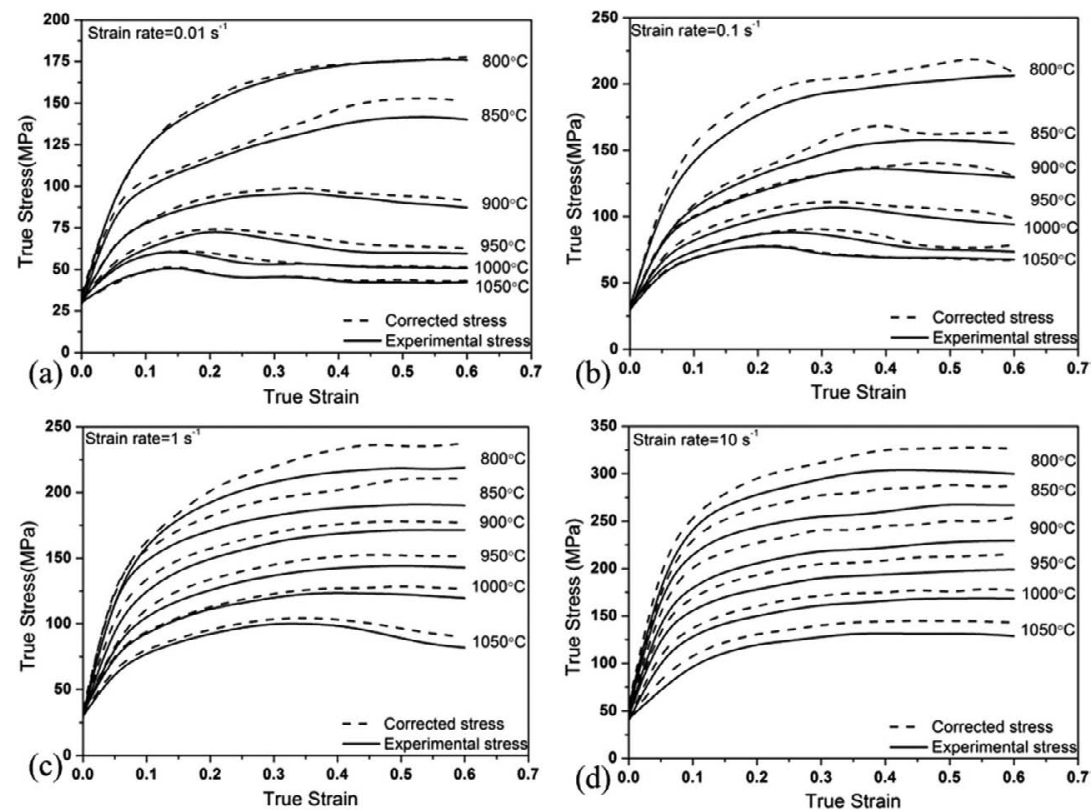

Fig. 3. True stress-true strain curves (corrected and experimental) at different temperatures and at strain rates of (a) $0.01 \mathrm{~s}^{-1}$, (b) $0.1 \mathrm{~s}^{-1}$, (c) $1 \mathrm{~s}^{-1}$ and (d) $10 \mathrm{~s}^{-1}$.
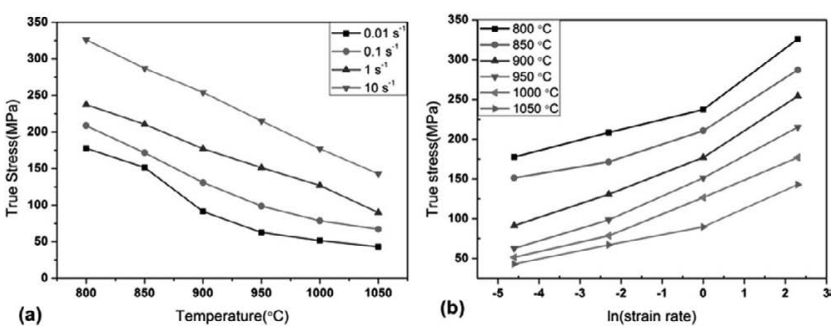

Fig. 4. Flow stress variation of the steel with (a) temperature, and (b) strain rate at true strain of 0.6 .

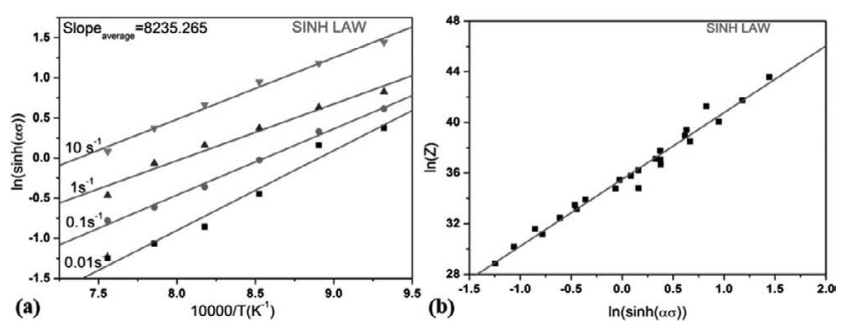

Fig. 6. Linear relationship between (a) $\ln [\sinh (\alpha \sigma)]$ vs $1 / \mathrm{T}$, and (b) $\ln (\mathrm{Z})$ vs $\ln (\sinh (\alpha \sigma))$.

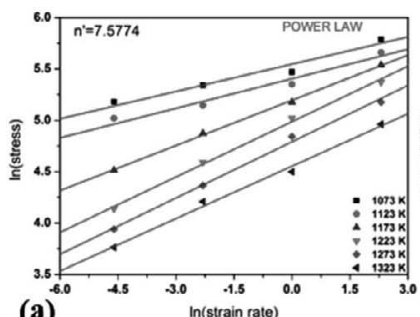

(a)

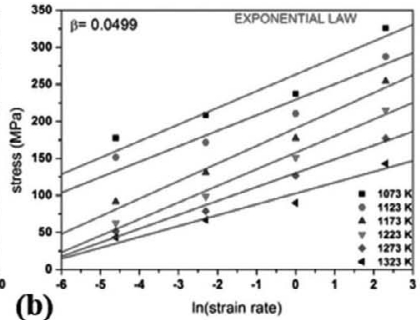

(b)

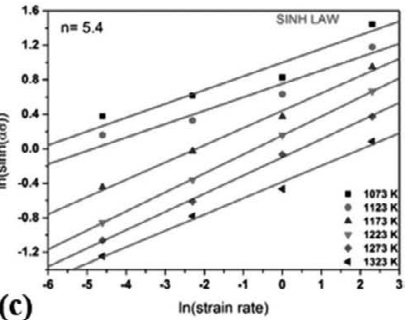

(c)

Fig. 5. Variation of strain rates with; (a) $\ln (\sigma)$, (b) stress and (c) $\ln [\sinh (\alpha \sigma)]$. 
the following constitutive relation (Eq. (15)) is obtained:

$$
Z=\dot{\varepsilon} \exp \left(\frac{368.1 \times 10^{3}}{R T}\right)=2.619 \times 10^{15}[\sinh (0.006598 \sigma)]^{5.4}
$$

\subsection{Flow Stress Prediction and Compensation of Strain}

It is assumed that there is negligible effect of strain on high temperature flow behavior and hence not considered in Eq. (12). ${ }^{15)}$ The material constants $(\beta, \alpha, n, Q$ and $\ln (A))$ of the constitutive relations are calculated for deformation strains ranging $0.05-0.6$ at an interval of 0.05 by the same procedure as described in above section for 0.6 strain. It has been observed that $\beta, \alpha, n, Q$ and $\ln (A)$ show significant variation with strain (Fig. 7). Coefficients $\beta, \alpha$, and $n$ are decreasing exponentially whereas activation energy and $\mathrm{ln}$ $(A)$ are increasing with increasing true strain. The activation energy increases from $311 \mathrm{kJmol}^{-1}$ at strain of 0.1 to 368 $\mathrm{kJmol}^{-1}$ at strain of 0.6 . Assuming these material constants as polynomial function of strain, and are fitted by varying degree of polynomial from 3 to 6 . A 5-degree polynomial (Eq. (16)), has best fit to the calculated data as shown in

(a)
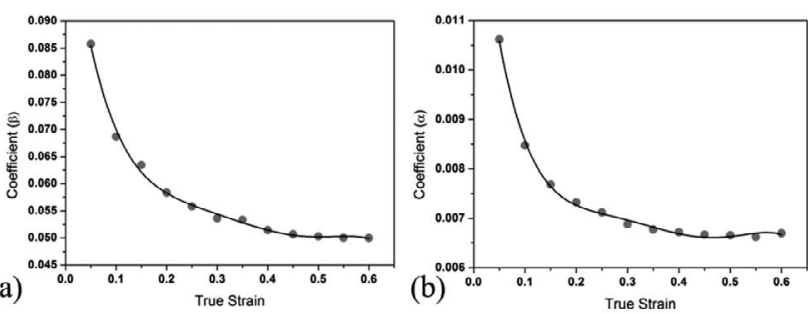

(c)
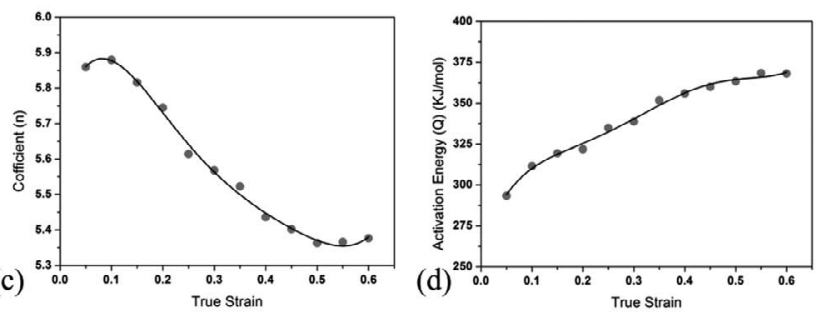

(e)

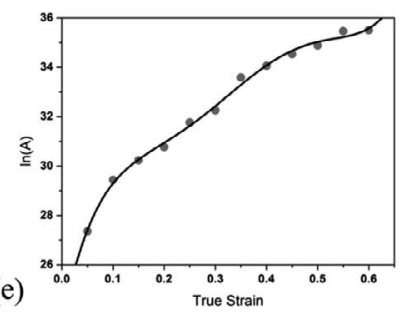

Fig. 7. Relationships between true strain and (a) $\beta$; (b) $\alpha$; (c) $n$; (d) $Q ;($ e) $\ln (A)$ by polynomial fit for $43 \mathrm{CrNi}$ steel.
Fig. 7. The values of coefficients of polynomial fitting of $\beta$, $\alpha, n, Q$, and $\ln (A)$ of the present steel are given in Table 2 . According to sinh type law, from Eq. (15), the flow stress $(\sigma)$ as a function of Zener-Hollomon parameter $(Z)$, can be given by Eq. (17).

$$
\begin{aligned}
\beta & =B_{0}+B_{1} \varepsilon+B_{2} \varepsilon^{2}+B_{3} \varepsilon^{3}+B_{4} \varepsilon^{4}+B_{5} \varepsilon^{5} \\
\alpha & =C_{0}+C_{1} \varepsilon+C_{2} \varepsilon^{2}+C_{3} \varepsilon^{3}+C_{4} \varepsilon^{4}+C_{5} \varepsilon^{5} \\
n & =D_{0}+D_{1} \varepsilon+D_{2} \varepsilon^{2}+D_{3} \varepsilon^{3}+D_{4} \varepsilon^{4}+D_{5} \varepsilon^{5} \ldots \ldots \\
Q & =E_{0}+E_{1} \varepsilon+E_{2} \varepsilon^{2}+E_{3} \varepsilon^{3}+E_{4} \varepsilon^{4}+E_{5} \varepsilon^{5} \\
\ln (A)= & F_{0}+F_{1} \varepsilon+F_{2} \varepsilon^{2}+F_{3} \varepsilon^{3}+F_{4} \varepsilon^{4}+F_{5} \varepsilon^{5} \\
& \sigma=\frac{1}{\alpha} \ln \left\{\left(\frac{Z}{A}\right)^{1 / n}+\left[\left(\frac{Z}{A}\right)^{2 / n}+1\right]^{1 / 2}\right\} \ldots \ldots \ldots . .
\end{aligned}
$$

4.4.1. Validation of the Established Constitutive Relation

With the intention to verify the determined constitutive equations for the present steel under hot deformation at high temperatures, the experimental and predicted stresses are compared (Fig. 8). ${ }^{18}$ Based on the above obtained values of material constants and activation energy at different strains between $0.05-0.6$ at interval of 0.05 , the values of flow stress are calculated for strain rates ranging from $0.01 \mathrm{~s}^{-1}-10 \mathrm{~s}^{-1}$ and the deformation temperatures ranging from $800^{\circ} \mathrm{C}-1050^{\circ} \mathrm{C}$ at an interval of $50^{\circ} \mathrm{C}$ using Eq. (17). It can be observed from the Fig. 8 that the developed constitutive equations have very good predictability of the flow stress with experimental stress.

\subsection{Microstructural Evolution}

The undeformed specimen was prepared by heating the as received steel specimen to a temperature of $1100^{\circ} \mathrm{C}$, holding for 2 minutes followed by water quenching. The optical micrograph of undeformed steel specimen is shown in Fig. 9. It consists of martensite needles as dark regions and proeutectoid ferrite as white regions. Small amount of retained austenite appearing as white region is associated with martensite needles. The optical micrographs of simulated hot deformed specimens at different given temperature and strain rate domain are shown in Figs. 10-12. The simulated deformation specimens (above $\mathrm{A}_{\mathrm{r} 3}$ ) show dark regions as martensite and bright regions as ferrite (shown by arrows). There is no pro-eutectoid ferrite before deformation due to complete austenitization and the entire ferrite was mainly formed during and/or after hot deformation. The ferrite phase formed may be strain induced and/or produced during cooling. The dark phase has average micro-hardness

Table 2. Results of 5 degree polynomial fit coefficients for $\beta, \alpha, n, Q$ and $\ln (A)$.

\begin{tabular}{crrrrrrrrr}
\hline$\beta$ & & $\alpha$ & & $n$ & & \multicolumn{3}{c}{$\ln (A)$} \\
\hline $\mathrm{B}_{0}$ & 0.11383 & $\mathrm{C}_{0}$ & 0.01452 & $\mathrm{D}_{0}$ & 5.69566 & $\mathrm{E}_{0}$ & 261.996 & $\mathrm{~F}_{0}$ & 23.63513 \\
$\mathrm{~B}_{1}$ & -0.74227 & $\mathrm{C}_{1}$ & -0.10441 & $\mathrm{D}_{1}$ & 5.38216 & $\mathrm{E}_{1}$ & 873.4672 & $\mathrm{~F}_{1}$ & 103.0235 \\
$\mathrm{~B}_{2}$ & 4.0079 & $\mathrm{C}_{2}$ & 0.59776 & $\mathrm{D}_{2}$ & -48.9446 & $\mathrm{E}_{2}$ & -5583.8055 & $\mathrm{~F}_{2}$ & -652.618 \\
$\mathrm{~B}_{3}$ & -11.1358 & $\mathrm{C}_{3}$ & -1.7049 & $\mathrm{D}_{3}$ & 154.1774 & $\mathrm{E}_{3}$ & 19485.0660 & $\mathrm{~F}_{3}$ & 2207.86 \\
$\mathrm{~B}_{4}$ & 15.11325 & $\mathrm{C}_{4}$ & 2.34782 & $\mathrm{D}_{4}$ & -223.242 & $\mathrm{E}_{4}$ & -30979.4865 & $\mathrm{~F}_{4}$ & -3424.29 \\
$\mathrm{~B}_{5}$ & -7.90615 & $\mathrm{C}_{5}$ & -1.23988 & $\mathrm{D}_{5}$ & 124.8071 & $\mathrm{E}_{5}$ & 17993.8009 & $\mathrm{~F}_{5}$ & 1954.242 \\
\hline
\end{tabular}



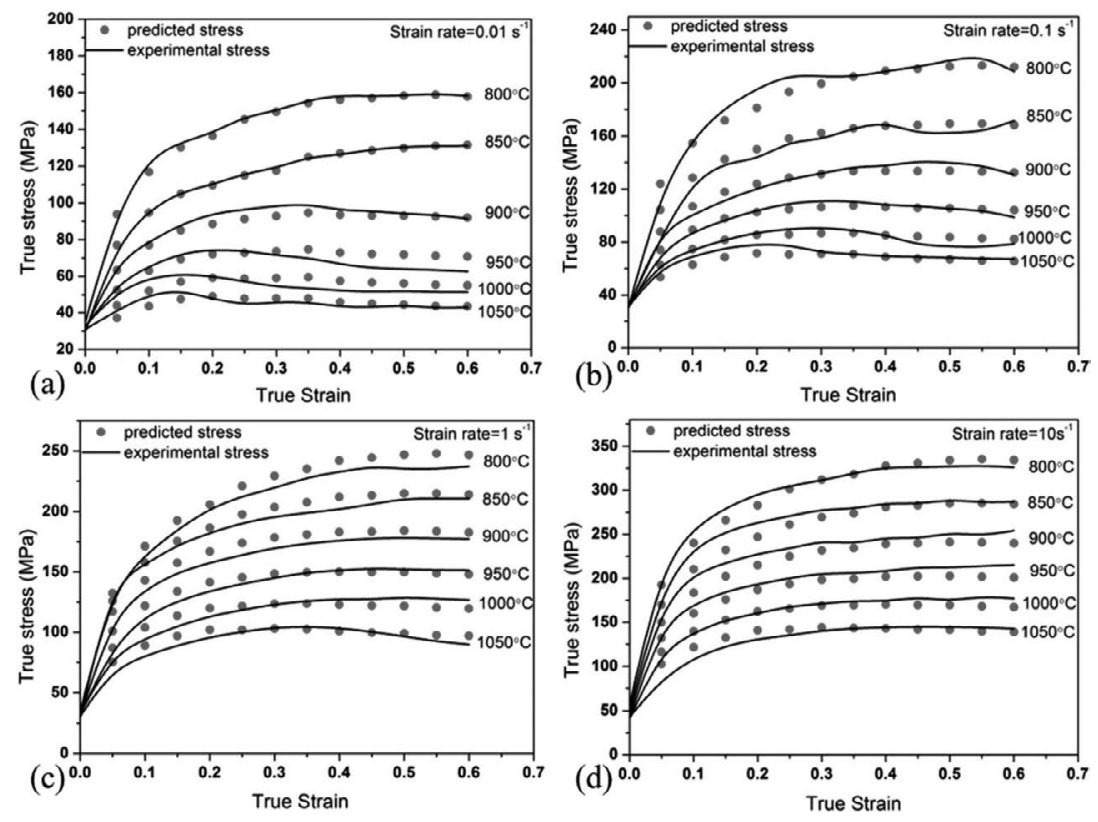

Fig. 8. Plots showing the experimental and predicted flow stresses at strain rate; (a) $0.01 \mathrm{~s}^{-1}$; (b) $0.1 \mathrm{~s}^{-1}$; and (c) $1 \mathrm{~s}^{-1}$ and (d) $10 \mathrm{~s}^{-1}$ in the temperature domain $800^{\circ} \mathrm{C}-1050^{\circ} \mathrm{C}$.

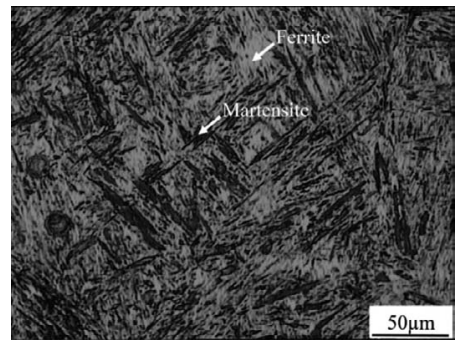

Fig. 9. Optical micrograph of undeformed steel specimen.
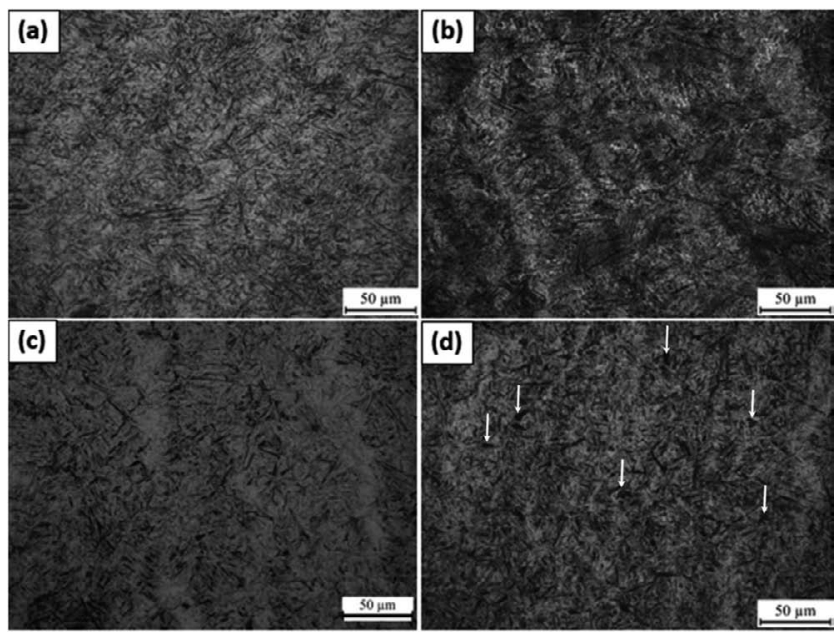

Fig. 10. Optical Micrographs of hot deformed steel specimens at (a) $800^{\circ} \mathrm{C}, 0.01 \mathrm{~s}^{-1}$; (b) $800^{\circ} \mathrm{C}, 10 \mathrm{~s}^{-1}$; (c) $900^{\circ} \mathrm{C}, 0.01 \mathrm{~s}^{-1}$ and (d) $900^{\circ} \mathrm{C}, 10 \mathrm{~s}^{-1}$.

of $760 \pm 10 \mathrm{VHN}$ which is in agreement with the Vickers hardness of martensite containing $0.4 \% \mathrm{C}^{23)}$ At temperature $800^{\circ} \mathrm{C}$, the pro-eutectoid ferrite is finer and elongated in shape at lower strain rate $\left(0.01 \mathrm{~s}^{-1}\right)$ (Fig. 10(a)) and its amount decreases with increasing strain rate $\left(10 \mathrm{~s}^{-1}\right)$ because of lower nucleation rate due to short deformation time. Small grain size and higher stored energy is favorable
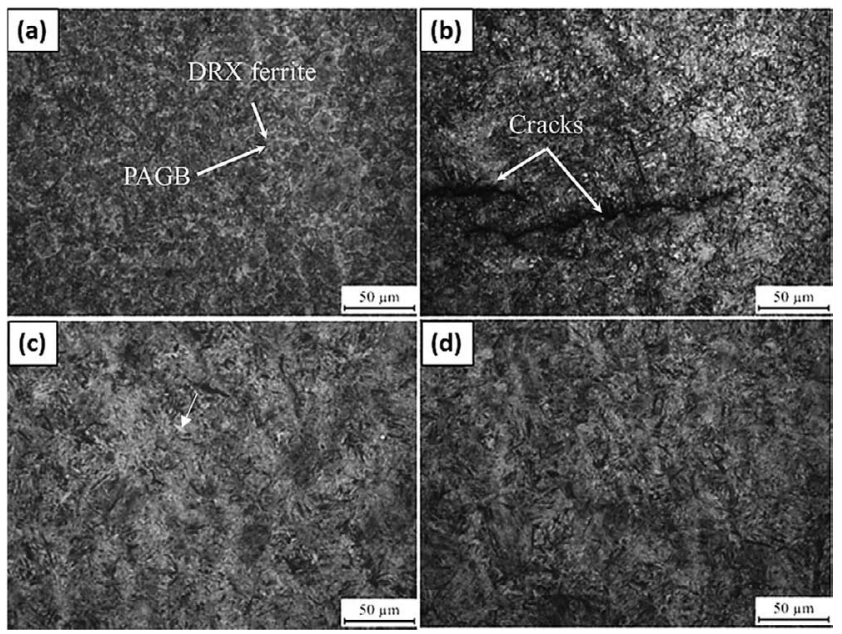

Fig. 11. Optical Micrographs of hot deformed steel specimens at (a) $1000^{\circ} \mathrm{C}, 0.01 \mathrm{~s}^{-1}$; (b) $1000^{\circ} \mathrm{C}, 10 \mathrm{~s}^{-1}$; (c) $1050^{\circ} \mathrm{C}$, $0.01 \mathrm{~s}^{-1}$ and (d) $1050^{\circ} \mathrm{C}, 10 \mathrm{~s}^{-1}$.

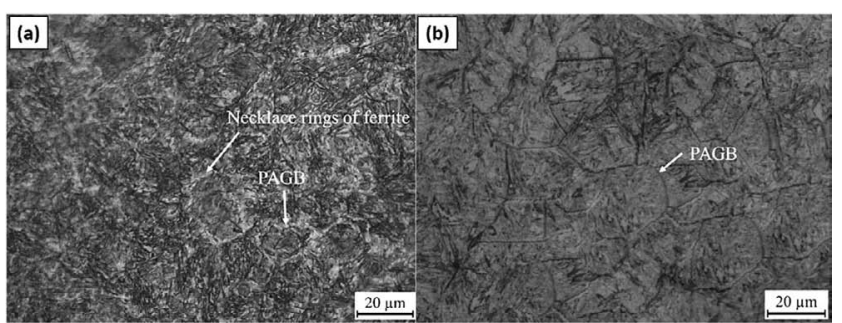

Fig. 12. Optical micrographs of simulated hot deformed steel specimens at (a) $1000^{\circ} \mathrm{C}$ and $0.1 \mathrm{~s}^{-1}$ showing ferrite necklace and (b) $1000^{\circ} \mathrm{C}$ and $0.1 \mathrm{~s}^{-1}$ showing PAGB

for the deformation induced ferrite transformation (DIFT). ${ }^{24)}$ Elongated pro-eutectoid ferrite appears along the martensitic structure due to incomplete recrystallization at high strain rates. At $900^{\circ} \mathrm{C}$, the ferrite volume fraction is $34 \%$ due to DIFT (Figs. 10(c) and 10(d)). The optical micrograph at deformation temperature $1000^{\circ} \mathrm{C}$ with strain rate $0.01 \mathrm{~s}^{-1}$ 
shows ferrite in the shape of necklace due to large amount of DRX (Fig. 11(a)). ${ }^{25)}$ Cracks have been observed in Fig. 11 (b). At high temperature deformation $\left(1050^{\circ} \mathrm{C}\right)$ and lower strain rate $\left(0.01 \mathrm{~s}^{-1}\right)$, some small blocky ferrite has formed during cooling as shown by arrow in Fig. 11(c). This blocky ferrite disappears at higher strain rate (Fig. 11(d)). The strain induced ferrite at the prior austenite grain boundary (PAGB) appearing in the form of necklace at $1000^{\circ} \mathrm{C}$ temperature and low strain rate $\left(0.1 \mathrm{~s}^{-1}\right)$ is shown in Fig. 12(a) and its PAGB is shown in Fig. 12(b). Prior austenite grain size (PAGS) has been found to decrease with increase in strain rate for a given temperature. At $900^{\circ} \mathrm{C}$, the PAGS has been found to vary from $36 \mu \mathrm{m}$ to $31 \mu \mathrm{m}( \pm 4 \mu \mathrm{m})$ as strain rate increases from 0.01 to $10 / \mathrm{s}$. This decrease in PAGS from 36 to $31 \mu \mathrm{m}$ is not a very significant change. However, it has been observed in this study that with increase in strain rate at a given temperature, PAGS is found to decrease. It is explained on the basis of shorter duration of dynamic recrystallization and growth of austenite grains (PAGS). However, for a given strain rate, decrease in temperature causes PAGS to increase during cooling. For example, at 0.01 strain rate, the PAGS increase from 25 to $46 \mu \mathrm{m}$ as temperature decreases.

\subsection{Hardness}

The variation of hardness values of various deformed specimens with different strain rates and temperatures is shown in Fig. 13. It can be observed that for a given temperature, hardness increases with increase in strain rate. This is attributed to increase in strain hardening (due to increase in dislocation density) in the specimens. For $1050^{\circ} \mathrm{C}$, hardness increases from $730 \mathrm{HV}$ to $770 \mathrm{HV}$ as strain rate increases from 0.01 to $10 \mathrm{~s}^{-1}$. For $800^{\circ} \mathrm{C}$, hardness varies from $798 \mathrm{HV}$ to $862 \mathrm{HV}$ for same range of increase in strain rate. However, for $900^{\circ} \mathrm{C}$ and $950^{\circ} \mathrm{C}$, a decrease in hardness with increase in strain rate at $1 \mathrm{~s}^{-1}$ is observed. Further it can be observed that for a given strain rate, increase in temperature decreases hardness of the specimens. This can be explained from the fact that higher temperature of compression causes more recrystallization resulting in decrease in hardness.

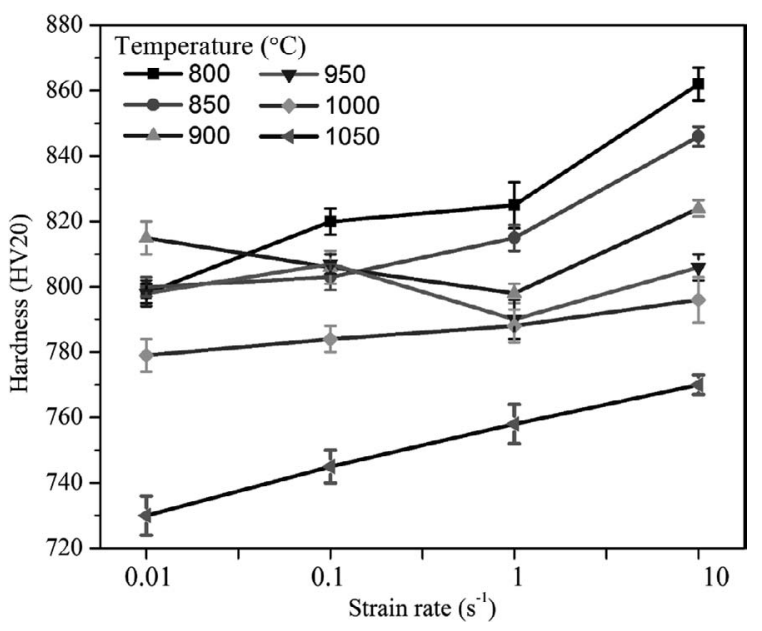

Fig. 13. Variation of hardness with strain rates and temperatures of hot deformed steel specimens.

\subsection{Processing Maps and Microstructural Correlation}

Processing map is the superimposition of power dissipation efficiency map and the instability parameter map. The instability parameter is shown by shaded area whereas efficiency is shown by iso-efficiency lines. The flow stress data obtained from the hot compression tests are used to plot the processing maps using different DMM models. These maps can give the processing window along with an opinion of deformation mechanisms that can be helpful for the industrial applications. To avoid accumulation of internal energy, the processing maps are plotted for strains after the strain hardening. ${ }^{26)}$ The flow stress data to plot processing maps at true strain of 0.3 and 0.6 are obtained from the flow curves at constant temperatures and strain rates. The strain rate sensitivity (Eq. (2)), power dissipation efficiency (Eqs. (5) and (8)) and instability parameter (Eqs. (6), (9) and (10)) of different models are calculated and plotted in the axes of $\log$ (strain rate) and temperature. Processing maps of true strain 0.3 and 0.6 are shown in Figs. 14 and 15. No instability is predicted when $D=P$, as it shows all
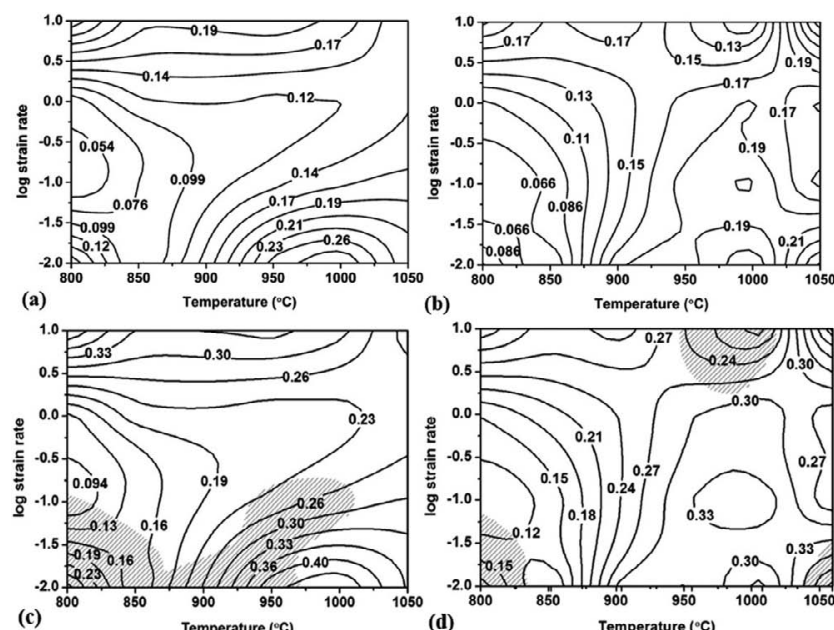

(b)
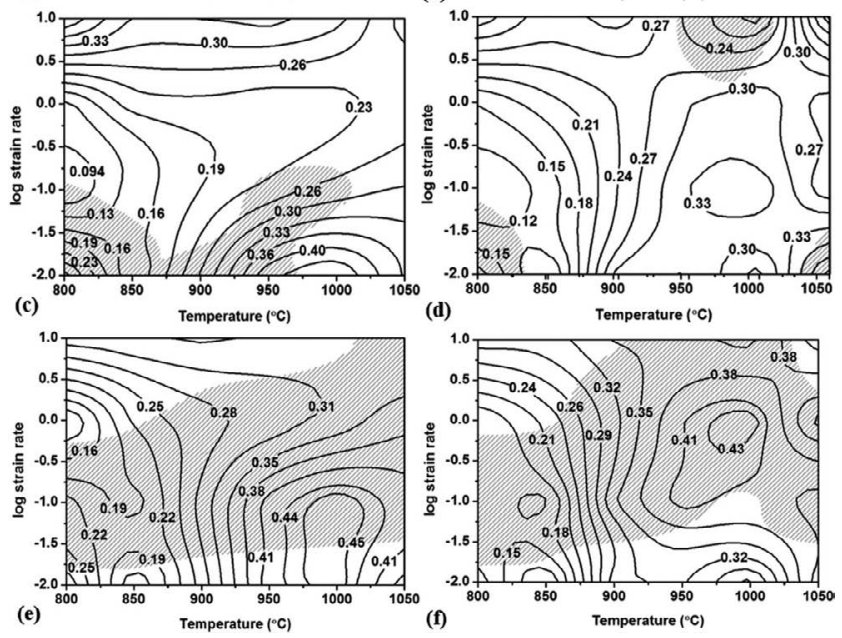

Fig. 14. Strain rate sensitivity $(m)$ maps of the steel at strain (a) $0.3 \&$ (b) 0.6; processing maps of the steel based on DMM of Prasad et al. at strain (c) $0.3 \&$ (d) 0.6 ; and processing maps based on modified DMM of Murty and Rao at strain (e) 0.3 , and (f) 0.6
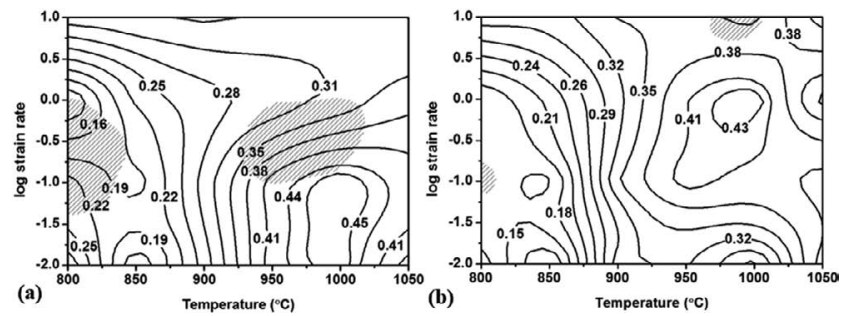

Fig. 15. Processing maps for the steel based on modified DMM and instability parameter $\kappa_{\mathrm{j}}$, at strain of (a) $0.3 \&$ (b) 0.6 . 
positive values of $m$ in the entire test zone. Large values of $m$ are observed at moderate to high temperature at almost all strain rates. Moreover, in this domain the value of stress increases steadily to a peak followed by softening towards the steady state region (Fig. 3), typically observed during DRX. ${ }^{27)}$ This is also proved by optical micrograph as shown in Fig. 11(c) having recrystallized grains without any cracks. It is because of no flow lines of the plastic deformation is observed. In addition, with increasing the strain rates and/ or decreasing the temperatures, these peaks are shifted towards higher strains (Figs. 3(a)-3(c)). Thus, the tendency of nucleation and growth of new grains is reduced with decrease in temperature or increase in strain rates (i.e. at high $Z$ value). ${ }^{28)}$ In these range, the values of $m$ and $\eta$ are high, and the stability is predicted. Whereas, at moderate temperatures and for almost all strain rates, the refinement of austenite grains takes place which is characterized by moderate values of $m$ and $\eta$. At low temperature and high strain rate, self-diffusion is retarded drastically. The value of $m$ increases as the deformation temperature increases for all strain rates. The iso-efficiency lines of $\eta_{\text {Prasad }}$ and the instability parameter $(\xi)$ are superimposed which yields processing maps (Figs. 14(c) and 14(d)). It can be observed that there exists following three zones:

1. A lower efficiency zone in the temperature range of $800^{\circ} \mathrm{C}-900^{\circ} \mathrm{C}$ and strain rate range of $0.01-3 \mathrm{~s}^{-1}$ with maximum efficiency of $24 \%$.

2. A peak zone between $875^{\circ} \mathrm{C}-1050^{\circ} \mathrm{C}$ and strain rate ranging from $0.01-3 \mathrm{~s}^{-1}$ having efficiency not less than $27 \%$.

3. Another peak zone in the temperature range of $1025^{\circ} \mathrm{C}-1050^{\circ} \mathrm{C}$ and strain rate ranging from $3-10$ $\mathrm{s}^{-1}$ having also efficiency not less than $30 \%$.

The values $\eta_{\text {Prasad }}$ follow the same trend as the $m$ values. The higher values of $\eta$ domains shift towards higher temperatures with the increasing strain. The iso-efficiency $\left(\eta_{\text {Prasad }}\right)$ directly related to the strain rate sensitivity parameter, $m$, whereas the correlation of iso-efficiency described by Murty and Rao ( $\eta_{\text {Murty and Rao }}$ ) and $m$ is insignificant. The iso-efficiency ( $\left.\eta_{\text {Murty and Rao }}\right)$ lines and the instability $(\kappa)$ domains (shaded area) are superimposed as shown in Figs. 14(e) and 14(f). High values of $\eta_{\text {Murty and Rao are observed at }}$ high temperatures, and low and high strain rate domains. From Fig. 16(a), pores (shown by arrow) can be observed in the microstructure, which is supported by the low values of efficiency and instability region. The flow stress behavior corresponding to Fig. 16 (a) $\left(800^{\circ} \mathrm{C}, 0.01 / \mathrm{s}\right)$ shows no dynamic recrystallization (DRX). Maximum flow stress

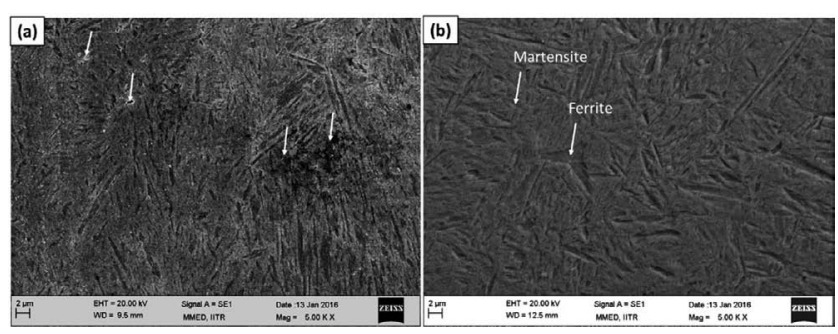

Fig. 16. SEM micrographs of steel specimen after hot compression at (a) $800^{\circ} \mathrm{C}, 0.01 \mathrm{~s}^{-1}$ (b) $950^{\circ} \mathrm{C}, 0.01 \mathrm{~s}^{-1}$. Pores are observed (shown by arrows). value is approximately $170 \mathrm{MPa}$. In Fig. $16(\mathrm{~b})\left(950^{\circ} \mathrm{C}\right.$, $0.01 / \mathrm{s}$ ), microstructure consists of ferrite and martensite, which is defect free complete recrystallized grains and lies at moderate efficiency and stable region. Maximum flow stress observed is approximately $73 \mathrm{MPa}$. This is also confirmed by the flow behavior of the specimen $\left(1050^{\circ} \mathrm{C}\right.$, $0.01 \mathrm{~s}^{-1}$ and $1050^{\circ} \mathrm{C}, 10 \mathrm{~s}^{-1}$ ) which shows the restoration phenomena likely to be DRV and DRX (Figs. 11(c) and $11(\mathrm{~d})$ ). Instability area predicted by $\kappa$ is larger than that of $\xi$. The values of $\eta_{\text {Murty and Rao }}$ increase with strains and shift towards higher temperature. Figs. 15(a) and 15(b) depict the iso-efficiency lines of $\eta_{\text {Murty and Rao }}$ and instability $\kappa_{j}$ domains. The instability zones predicted by $\kappa_{j}$ are smaller than those predicted by $\kappa$ either or $\xi$, whereas the region represented by $\eta_{\text {Murty and Rao }}$ is almost similar to that of $\eta_{\text {Prasad }}$ and strain rate sensitivity parameter $(m)$. This advocates that the above parameters are closely related with the microstructural behaviour, and diffusive process governs the deformation mechanisms in visco-plastic materials. The instability parameter, $\kappa_{j}$, proposed by Poletti et al. is directly related to the dissipative function which is associated to microstructural changes, $J$, whereas the instability parameters, $\xi$ and $\kappa$, proposed by Prasad et al. and Murty and Rao, respectively, are associated with the strain rate sensitivity parameter, $m$. Therefore, the instability parameter, $\kappa_{j}$, is more mathematically precise as it eliminates $m$ from its calculations.

In general, the value of $m$ and $\eta$ increases with increase in temperatures for almost all strain rates as given by processing maps of different models (Figs. 14 and 15). At low strain rates, large values of $m$ and $\eta$, and smaller apparent activation energy are observed, which are related to diffusion controlled processes resulting in better workability. At high temperatures diffusion process takes place which is related to the DRX of austenite. Flow softening arises locally during hot working at high strain rates due to adiabatic heating, which results in defects like formation of voids/pores and cracks in the specimen. This is confirmed by the specimen deformed at deformation temperature $1000^{\circ} \mathrm{C}$ with strain rate of $10 \mathrm{~s}^{-1}$ (Fig. 11(b)). This damage in the specimen is due to the short time of deformation (at high strain rates) because of less restoration phenomena such as DRX and dynamic recovery. Small voids/cracks are also present in the microstructure of the specimens deformed at $10 / \mathrm{s}, 900^{\circ} \mathrm{C}$ as shown by arrow in the Fig. 10(d). This is due to the fact that the deformation zone is in the higher efficiency and stable region as shown in Fig. 14(d) as compared specimen at $10 / \mathrm{s}, 1000^{\circ} \mathrm{C}$. The low values of strain rate sensitivity parameter $m$, also support the less restoration of microstructure (appearance of pores). This is also confirmed by the specimen deformed at deformation temperature of $800^{\circ} \mathrm{C}$ with strain rate of $0.01 \mathrm{~s}^{-1}$ (Fig. 16(a)).

\section{Conclusions}

The main conclusions of the present investigation are summarized below:

(1) The experimental true stress-true strain data in temperature range $800^{\circ} \mathrm{C}-1050^{\circ} \mathrm{C}$ and strain rate range $0.01-10 \mathrm{~s}^{-1}$, are analyzed by comprehensive application of different approaches comprising constitutive equation analysis, processing maps and microstructural correlation. 
(2) The experimental stresses are corrected for adiabatic heating which is substantial at higher strain rates.

(3) The developed constitutive equation for predicting flow stresses are verified by comparing with experimental stresses, and found to be having good correlation coefficient $\left(R^{2}=0.98\right)$. The errors between the measured and calculated values are less than $6.5 \%$ for the present steel in all cases. The constitutive equation of the investigated steel at true strain of 0.6 can expressed as follows:

$$
Z=\dot{\varepsilon} \exp \left(\frac{368.1 \times 10^{3}}{R T}\right)=2.619 \times 10^{15}[\sinh (0.006598 \sigma)]^{5.4}
$$

(4) The flow curves demonstrate the DRX behavior with a single stress peak followed by softening towards the steady state in wide range of temperatures and strain rates. However, at high temperature and low strain rates, curves display cyclic DRX behavior having multiple peaks.

(5) The apparent activation energy of hot deformation using sinh type law is found to be $368 \mathrm{kJmol}^{-1}$ at true strain of 0.6 for the present steel. The value of stress exponent $n$ is 5.4 using sinh type equation, which suggests that the mechanism of hot deformation is dislocation glide controlled by dislocation climb.

(6) High values of $m$ and $\eta$ at low strain rates, and moderateto high temperatures $\left(950-1050^{\circ} \mathrm{C}\right)$ result from DRX of austenite. A moderate values of $m$ and $\eta$ was observed at moderate temperatures, and low to moderate strain rates, due to result of strain induced ferrite formation and dynamic recovery through stable flow.

(7) The efficiency of power dissipation $\eta$ and the instability domain vary with the increasing strain. Metallographic investigations show that the damage at high strain rates occur by a combination of limited restoration of microstructure due to short deformation times and possible adiabatic heating.

\section{Acknowledgments}

The authors greatfully wish to acknowledge the Department of Science and Technology (DST) New Delhi, Government of India for purchasing the Thermo mechanical simulator in IIT Roorkee from the FIST grant (SR/FST/ ETI-216/2007 Dated 06.02.2008).

\section{REFERENCES}

1) Y. C. Lin, M. S. Chen and J. Zhong: J. Mater. Process. Technol., 205 (2008), 308

2) H. Zhao, G. Liu and L. Xu: Mater. Sci. Eng. A, 559 (2013), 262

3) Y. C. Lin, M. S. Chen and J. Zhang: Mater. Sci. Eng. A, 499 (2009), 88.

4) K. Hase and N. Tsuji: Scr. Mater., 65 (2011), 404.

5) H. Wei, G. Liu, H. Zhao and M. Zhang: Mater. Sci. Eng. A, 596 (2014), 112.

6) S. K. Rajput, M. Dikovits, G. P. Chaudhari, C. Poletti, F. Warchomicka, V. Pancholi and S. K. Nath: Mater. Sci. Eng. A, 587 (2013), 291.

7) V. Raghavan: Physical Metallurgy, Prentice Hall of India, New Delhi, (2008), 199.

8) Y. V. R. K. Prasad, H. L. Gegel, S. M. Doraivelu, J. C. Malas and J. T. Morgan: Metall. Trans. A, 15 (1984), 1883.

9) Y. V. R. K. Prasad: J. Mater. Eng. Perform., 12 (2003), 638.

10) H. Ziegler: Progress in Solid Mechanics, John Wiley and Sons, New York, (1963), 93.

11) S. Narayana Murty: Compos. Sci. Technol., 63 (2003), 119

12) C. Poletti, J. Six, M. Hochegger, H. P. Degischer and S. Ilie: Steel Res. Int., 82 (2011), 710.

13) S. K. Rajput, G. P. Chaudhari and S. K. Nath: J. Mater. Eng. Perform., 23 (2014), 2930.

14) K. K. Saxena, S. Sonkar, V. Pancholi, G. P. Chaudhari, D. Srivastava, G. K. Dey, S. K. Jha and N. Saibaba: J. Alloy. Compd., 662 (2016), 94.

15) D. Samantaray, S. Mandal and A. K. Bhaduri: Mater. Des., 31 (2010), 981.

16) J. Zhang, H. Di, K. Mao, X. Wang, Z. Han and T. Ma: Mater. Sci. Eng. A, 587 (2013), 110.

17) Y. V. R. K. Prasad, K. P. Rao and S. Sasidhara: Hot Working Guide: A Compendium of Processing Maps, ASM International, Materials Park, OH, (2015), 2.

18) L. X. Kong and P. D. Hodgson: ISIJ Int., 39 (1999), 991.

19) H. Mirzadeh, J. M. Cabrera, J. M. Prado and A. Najafizadeh: Mater. Sci. Eng. A, $\mathbf{5 2 8}$ (2011), 3876.

20) C. Zhang, L. Zhang, W. Shen, C. Liu, Y. Xia and R. Li: Mater. Des., 90 (2016), 804.

21) S. Sajadifar, G. Yapici, M. Ketabch and B. Bemanizadeh: J. Iron Steel Res. Int., 20 (2013), 133.

22) N. E. Dowling: Mechanical Behavior of Materials, Pearsons Prentice Hall, London, (2007).

23) V. Raghavan: Physical Metallurgy, Prentice Hall of India, New Delhi, (2008), 84.

24) Z. Yang and R. Wang: ISIJ Int., 43 (2003), 761.

25) H. Wei, G. Liu, X. Xiao and M. Zhang: Mater. Sci. Eng. A, 573 (2013), 215.

26) C. Poletti, H. Dieringa and F. Warchomicka: Mater. Sci. Eng. A, 516 (2009), 138.

27) E. I. Poliak and J. J. Jonas: ISIJ Int., 43 (2003), 684

28) S. M. Abbasi and A. Momeni: Mater. Sci. Eng. A, 552 (2012), 330. 